\title{
Dual-Halbach Array Permanent Magnet Tubular Generator for Free-Piston Generator
}

\author{
Hassan Moradi Cheshmeh Beigi ${ }^{1}$ and Sohrab Akbari ${ }^{2 *}$ \\ ${ }^{1}$ Electrical Engineering Department, Faculty of Engineering, Razi University, Kermanshah 67149, Iran \\ ${ }^{2}$ Electrical Power Distribution Company Kermanshah, Iran
}

(Received 24 June 2015, Received in final form 11 September 2015, Accepted 17 September 2015)

\begin{abstract}
In this study, we describe the effects of changing the magnet shape of permanent magnets (PMs) in a rotor Halbach-array PM generator for reciprocating free piston generator applications. More specifically, the rectangular-shaped magnets were replaced by the trapezoidal-shaped magnets. The initial design, an analytical magnetic field solution of rectangular shaped magnets, is presented and air-gap magnetic flux density and thrust force were estimated. The results were compared to the finite element analysis (FEA) showing excellent agreement. Using FEA, the effect of the shape of the magnets on the flux density and thrust force waveforms is analyzed. Moreover, the proportion of the Halbach array in the machine was optimized by the means of a parametric search. The results obtained from the analytical calculations and FEA were validated by comparing to those of Radial-array PM generator.
\end{abstract}

Keywords : Dual-Halbach Array, free piston generator, finite element analysis, parametric search.

\section{Introduction}

In this study, we describe the merits of changing the PM shape in Halbach arrays applied to tubular generators. These generators are increasingly used owing to their high efficiency, high power/force density, and down ripple force characteristics [1].

This class of machines is found to be suitable for reciprocating free piston generator applications such as in an improved output power and a voltage for free piston generator, warranting the utmost average thrust force and least value of cogging force [2]. Although a few core-type Halbach-array PMs [3, 4] have been reported, the possibility of a coreless configuration utilizing a dual Halbacharray PM has not been investigated in detail.

PM tubular generators have been proved to provide the best all-around performance for reciprocating free piston generator applications in comparison to induction-type generator and switched reluctance generator, because of their high force and high efficiency [5-7]. A radial array, which is a special case of PM generators, is less used for

CThe Korean Magnetics Society. All rights reserved.

*Corresponding author: Tel: +989336806831

Fax: +988334221063, e-mail: Sohrab.akbari7@yahoo.com reciprocating free piston generator applications, because of its high ripple force production. The magnitude of the detent force presented in the radial array is almost $13 \%$ of the developed force [8]. Core-type Halbach array PM generators also give rise to ripple force including the cogging force produced by the machine. Cogging is one of the disadvantages faced in the slotted generator design, as it causes a ripple in the force generated by the generator [9]. The advantages of slotless Permanent Magnet Tubular Generator (PMTG) have numerous attributes making them an ideal choice for a lot of applications [10]:

-Zero-cogging torque for smooth operation and minimal vibration.

- Excellent power-to-weight ratio enabling the design of compact, light-weight hand tools

\section{Dual Array PMTG}

A dual array PMTG utilizes a slotless stator winding unlike the conventional slotted-type PMTG. The concentrated type of winding is used owing to the advantage of no overlapping of phase windings. Being air cored, this machine possesses zero cogging thrust force. The introduction of horizontal magnetized magnet eliminates the flux through the rotor iron core, and hence the core loss. 


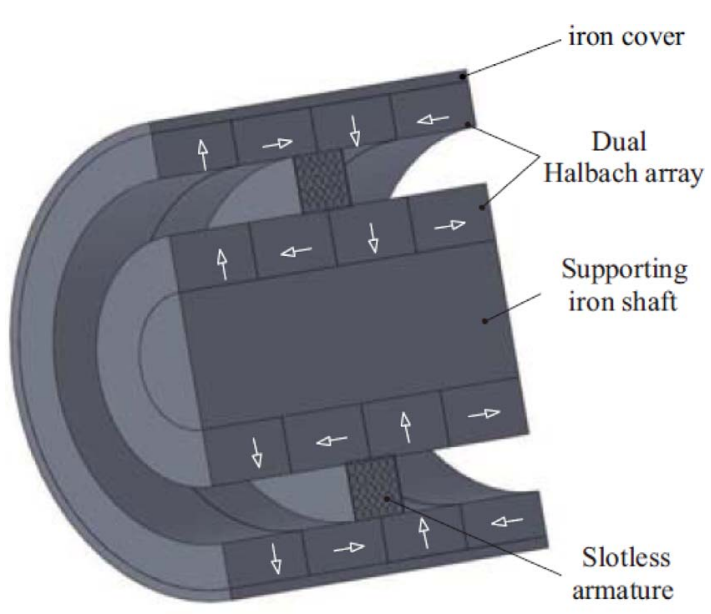

Fig. 1. PMTG with dual Halbach array.

In addition to that, the air-gap flux density also increases, resulting in high thrust force. With these advantages, this machine is an ideal candidate for low-speed high-force reciprocating free piston generator applications requiring output power of more than the conventional slotted-type PMTG. Figure 1 shows the PMTG with a dual Halbach array.

\section{Formulation of Magnetic Field and Thrust Force}

Magnetic field modeling is a precondition of thrust force formulation, and analytical field model is a powerful tool for designing and analyzing linear machines. From the Maxwell equation and magnetic property of different materials, the governing equations of magnetic field, i.e., Laplace's and Poisson's equations are significant for the solution of magnetic field. The general schematic of the machine shown in Fig. 2 is divided into two regions, in which region 2 is the Halbach magnetized magnets and the other region is air/winding region.

To establish an analytical solution for the field distribution produced in a multipole Halbach machine, the following assumptions were made:

1) The length of the machine is extended to infinity.

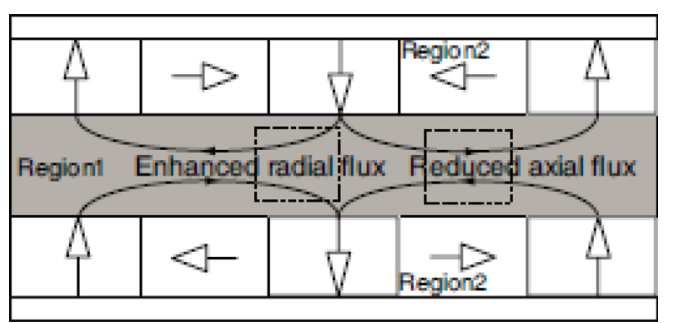

Fig. 2. (Color online) General schematic diagram of a coreless dual Halbach-array PMTG.
2) The back iron is infinitely permeable.

3) The permeability of PM material is equal to the permeability of free space.

Therefore, the governing field equations, in terms of magnetic vector potential, lead to Laplace and Poisson equations as follows [11]:

$$
\left\{\begin{array}{cc}
\nabla^{2} A_{1}=0 & \text { in region } 1 \\
\nabla^{2} A_{2}=-\mu_{0} J_{M} & \text { in region } 2 .
\end{array}\right.
$$

The vector potential $\mathrm{A}$ has only one component, $A(\theta)$, which is independent of $\theta$ in the cylindrical system. Therefore, Equations (1) and (2) can be written as

$$
\left\{\begin{array}{c}
\frac{\partial}{\partial z}\left(\frac{1}{r} \frac{1}{\partial z}\left(r A_{1 \theta}\right)\right)+\frac{\partial}{\partial r}\left(\frac{1}{r} \frac{1}{\partial r}\left(r A_{1 \theta}\right)\right)=0 \quad \text { in region 1, (3) } \\
\frac{\partial}{\partial z}\left(\frac{1}{r} \frac{1}{\partial z}\left(r A_{2 \theta}\right)\right)+\frac{\partial}{\partial r}\left(\frac{1}{r} \frac{1}{\partial r}\left(r A_{2 \theta}\right)\right)=-\mu_{0} J_{M} \text { in region 2. (4) }
\end{array}\right.
$$

The remanent magnetization vector can be written in the form of its components $M=M_{r} e_{r}+M_{z} e_{z}$, and the flux density $\mathrm{B}$ obtained from $\mathrm{A}$ is represented by $B_{z}=1 / r$. $\partial / \partial r\left(r A_{\theta}\right)$ and $B_{r}=\partial A_{\theta} / \partial_{z}$.

In the Fourier series form, the magnetization vector $M$ is decomposed into harmonics as shown by Equation (5).

$$
\left\{\begin{array}{l}
M_{r}=\sum_{n=1,2, \ldots}^{\infty} M_{r n} \sin m_{n} z, \\
M_{z}=\sum_{n=1,2, \ldots}^{\infty} M_{z n} \sin m_{n} z,
\end{array}\right.
$$

where

$$
\left\{\begin{array}{l}
m_{n}=\frac{(2 n-1) \pi}{2}, \\
M_{r n}=\frac{4 B_{r e m}}{\mu_{0} \tau_{p} m_{n}} \sin \frac{m_{n} \tau_{p}}{2} \sin \frac{m_{n} \tau_{m r}}{2}, \\
M_{z n}=\frac{4 B_{r e m}}{\mu_{0} \tau_{p} m_{n}} \sin \frac{m_{n} \tau_{m z}}{2} .
\end{array}\right.
$$

$\tau_{p}$ is the PM pole pitch and $B_{r}$ is the remanence. Therefore, Equation (2) can be written as

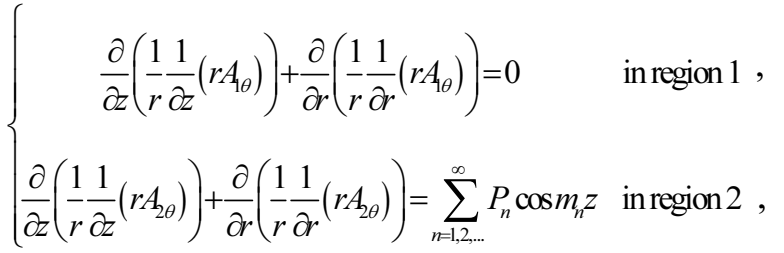

where 


$$
P_{n}=-\frac{4 B_{r e m}}{\tau_{p}} \sin \frac{m_{n} \tau_{p}}{2} \sin \frac{m_{n} \tau_{m r}}{2} .
$$

As a result, the general solutions to the Laplace and Poisson equations are:

$$
\begin{array}{cc}
A_{\theta 1}=\sum_{n=1,2, \ldots}^{\infty}\left(a_{1 n} B I_{1}\left(m_{n} r\right)+b_{1 n} B K_{1}\left(m_{n} r\right)\right) \cos \left(m_{n} z\right) & \text { Region 1, (10) } \\
A_{\theta 2}=\sum_{n=1,2, . .}^{\infty}\left(\left(a_{2 n} B I_{1}\left(m_{n} r\right)+b_{2 n} B K_{1}\left(m_{n} r\right)\right) \cos \left(m_{n} z\right)+S(r, z)\right) & \text { Region 2. (1 }
\end{array}
$$

Equation (11) is the general solution of Equation (8), which is a nonhomogeneous Bessel's differential equation. Therefore, Equation (11) contains Struve functions [12] that exist in the unknown term $S(r, z)$. Solving Equation (8) for $S(r, z)$ yields:

$$
S(r, z)=\frac{1}{2} \frac{\pi \text { StruveL }_{1}\left(m_{n} r\right) P_{n} \cos \left(m_{n} z\right)}{m_{n}^{2}},
$$

where Struve $L_{1}$ is the modified Struve function of order one. The flux distributions in all the two regions are derived from Equations (10) and (11), yielding to the following equations.

$$
\begin{aligned}
& B_{r 1}=\sum_{n=1,2, \ldots}^{\infty} m_{n}\left(a_{1 n} B I_{1}\left(m_{n} r\right)+b_{1 n} B K_{1}\left(m_{n} r\right)\right) \sin \left(m_{n} z\right) \text { Region 1, (13) } \\
& B_{z 1}=\sum_{n=1,2, \ldots}^{\infty} m_{n}\left(a_{1 n} B I_{0}\left(m_{n} r\right)-b_{1 n} B K_{0}\left(m_{n} r\right)\right) \cos \left(m_{n} z\right) \text { Region 1, (14) } \\
& B_{r 2}^{p}=\sum_{n=1,2, \ldots}^{\infty} m_{n}\left(a_{2 n}^{p} B I_{1}\left(m_{n} r\right)+b_{2 n}^{p} B K_{1}\left(m_{n} r\right)\right) \sin \left(m_{n} z\right)+S(r, z) \text { Region 2, (15) } \\
& B_{z 2}^{p}=\sum_{n=1,2, \ldots}^{\infty} m_{n}\left(a_{2 n}^{p} B I_{0}\left(m_{n} r\right)-b_{2 n}^{p} B K_{0}\left(m_{n} r\right)\right) \cos \left(m_{n} z\right)+S(r, z) \quad \text { Region 2, (16) }
\end{aligned}
$$

where $B I_{0}$ and $B I_{1}$ are the modified Bessel function of the first kind of order $1 ; B K_{0}$ and $B K_{1}$ are the modified Bessel function of the second kind of order 1 ; and $a_{n}$ and $b_{n}$ are constants. Equations (13) to (16) represent the field density of the whole machine. $B r 1$ and $B z 1$ represent the radial and axial magnetic field in the winding region, whereas $B_{r 2}^{p}$ and $B_{z 2}^{p}$ are in the magnetic regions. The upper script, $p=1 ; 2$, represents the external and internal $\mathrm{PMs}$, respectively. The boundary conditions to be satisfied by the solution to Equations (7) and (8) are:

$$
\begin{aligned}
& \left.B_{z 2}^{1}\right|_{r=R_{s}}=\mu_{0} M_{z}^{1},\left.\quad B_{z 2}^{2}\right|_{r=R_{r}}=\mu_{0} M_{z}^{2},\left.\quad B_{r 1}\right|_{r=R_{b}}=\left.B_{r 2}^{1}\right|_{r=R_{b}}, \\
& \left.H_{z 1}\right|_{r=R_{b}}=\left.H_{z 2}^{1}\right|_{r=R_{b}},\left.\quad B_{r 1}\right|_{r=R_{a}}=\left.B_{r 2}^{2}\right|_{r=R_{a}},\left.\quad H_{z 1}\right|_{r=R_{b}}=\left.H_{z 2}^{2}\right|_{r=R_{a}},
\end{aligned}
$$

The thrust force output of electromagnetic linear machine is governed by the Lorentz law. The current in the windings interacts with magnetic field to generate an axial thrust force represented by Equation (17).

$$
F_{w}=\int_{V}(J \times B) d V,
$$

where $J$ is the current density. Figure 3 shows the structure of a single phase machine. Each winding occupies an axial width of $\tau_{w}$. The two coils of one phase are separated by a distance of $\tau_{w p}$. The thrust force exerted on one coil is as follows.

$$
F_{W}=-4 \pi J \sum_{n=1}^{\infty} K_{r n} \sin \left(m_{n} \frac{\tau_{w}}{2}\right) \cos \left(m_{n} z\right)
$$

where $R_{i}$ is the outer radius of the armature. $K_{r n}$ is given by:

$$
K_{r n}=\int_{R_{a}}^{R_{i}} r\left[a_{1 n} I_{1}\left(m_{n} r\right)+b_{1 n} K_{1}\left(m_{n} r\right)\right] d_{r} .
$$

Therefore, the thrust force exerted on a single winding is represented by the following equation.

$$
F_{w p}=F_{w}(z)-F_{w}\left(z-\tau_{w p}\right)=8 \pi J \sum_{n=1}^{\infty} K_{n} \sin \left[m_{n}\left(z-\frac{\tau_{w p}}{2}\right)\right],
$$

where $K_{n}$ is given by

$$
K_{n}=\sin \left(m_{n} \frac{\tau_{w}}{2}\right) \sin \left(m_{n} \frac{\tau_{w p}}{2}\right) K_{r n} .
$$

Substituting $J=\sqrt{2} J_{r m s} \cos \omega t$ into equation (20) gives

$$
F_{w p}=-8 \sqrt{2} J_{r m s} \sum_{n=1}^{\infty} K_{n} \sin \left(m_{n}\left(z-\frac{\tau_{w p}}{2}\right)\right) \cos \omega t
$$

where $J_{r m s}$ is the root mean square of the current density; and $\tau_{w p}$ is the winding pitch. $R_{i}$ is defined as $R_{i}=R_{a}+g$, where $R_{a}$ is the outer radius of the internal PMs; $R_{b}$ is the inner radius of the external PMs; $g$ is the size of the air gap, and $\tau_{w}$ is the axial width of the phase winding per pole. Given $v \frac{T}{2}=\tau_{p}$, where $v$ is the velocity of the mover, following equation is obtained.

$$
\omega t=\frac{\pi v}{\tau_{p}} t=\frac{\pi}{\tau_{p}}(z-x),
$$

where $x$ and $z$ are the starting position and the current position of the phase winding, respectively. Equation (23) shows that the starting position and current position affect the instantaneous current and thus thrust force output. Substituting into Equation (22) yields the thrust force produced by a single-phase winding.

$$
F_{w p}=-8 \sqrt{2} J_{r m s} \sum_{n=1}^{\infty} K_{n} \sin \left(m_{n}\left(z-\frac{\tau_{w p}}{2}\right)\right) \cos \left[\frac{\pi}{\tau_{p}}(z-x)\right] .
$$




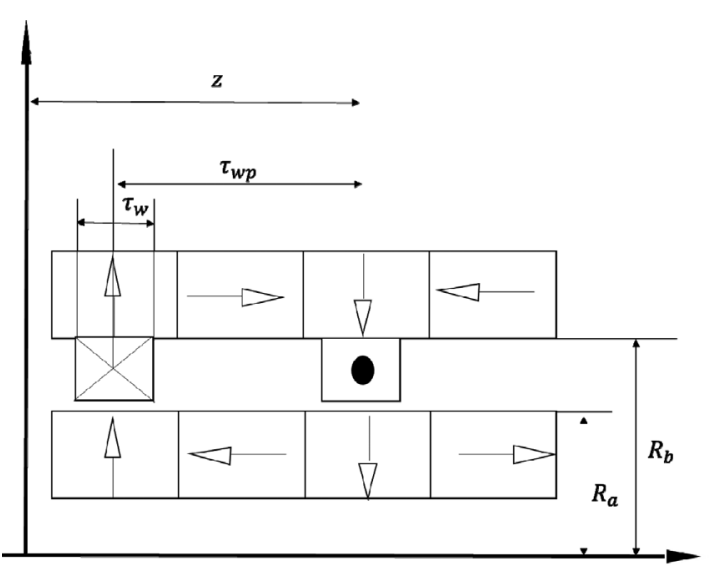

Fig. 3. Single-phase winding structure.

The machine thrust force is not only related to the current motion position $z$, but also the starting position $x$.

\section{Analytical Results}

The closed form solution derived in the previous section is used for computing the radial component of the air-gap flux density and thrust force for the rectangular shaped dual Halbach-array PMTG. Figure 4 shows the air-gap flux density variation of dual quasi-Halbach array and radial array, which was calculated by Equation [13]. The amplitude of the flux density of dual Halbach-array PMTG follows the higher amplitude obtained for the radial-array PMTG.

Thrust force is important for the analysis of machine performance. Based on the derived mathematical model, thrust force for a PM linear machine was studied. The result of the thrust force for dual quasi-Halbach array and radial array topologies is shown in Fig. 5. As shown, the

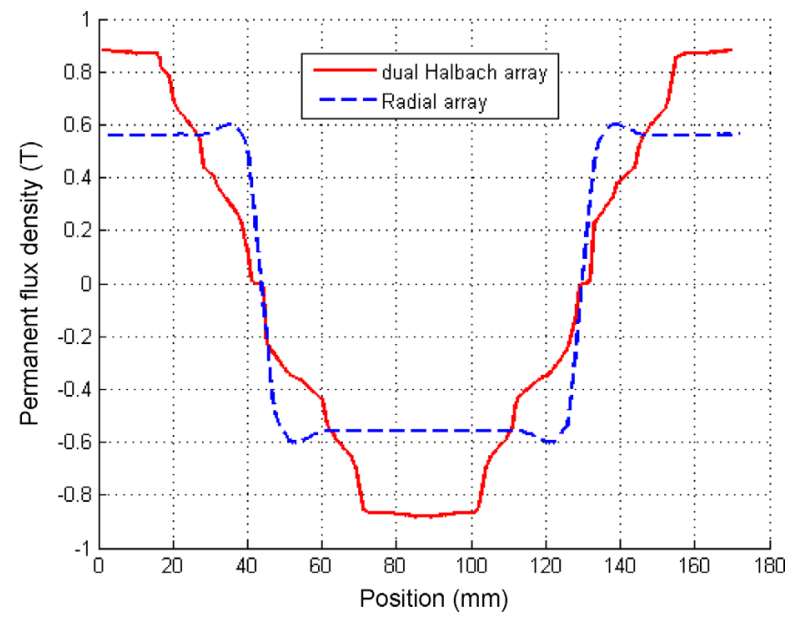

Fig. 4. (Color online) A comparison of the flux density of the dual Halbach array and radial array topologies.

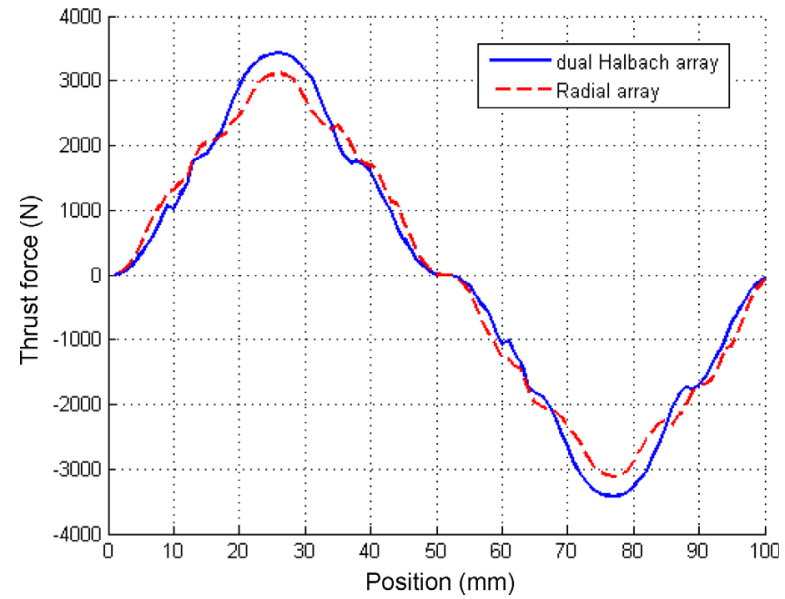

Fig. 5. (Color online) Thrust force of radial array and dual quasi-Halbach array topologies.

amplitude of the flux density of dual Halbach-array PMTG is higher than the amplitude obtained for the radialarray PMTG.

\section{FE Modeling and Analysis of the Machine}

\subsection{FE Modeling and Analysis}

A 2-D nonlinear time-stepping FE method was used to verify the accuracy of the results obtained from the analytical results. The imagery of the machine was carried out using a COMSOL software package. Figure 6 shows the flux lines in the PMTG obtained by the FE methods. Clearly, the horizontal magnetized magnet acts as a path for the flux between the adjacent poles and reduces the flux in the shaft. This effect will be clearer with an increased proportion between the horizontal and vertical magnetized magnets.

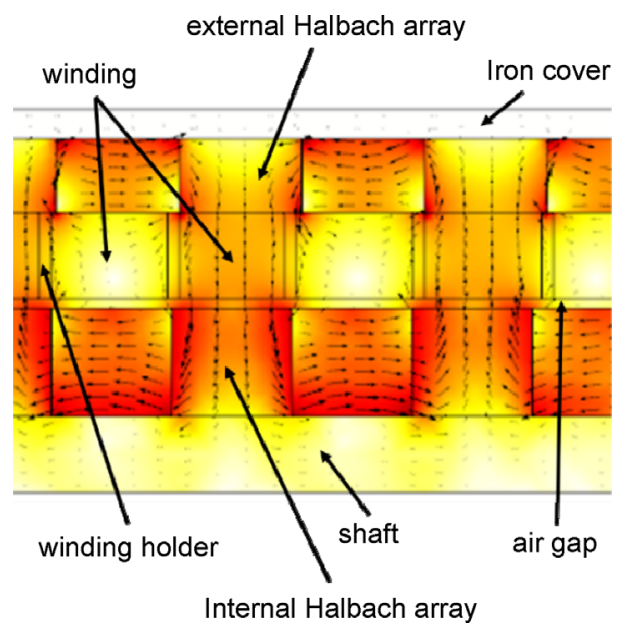

Fig. 6. (Color online) Flux distributions in dual quasi-Halbach array. 


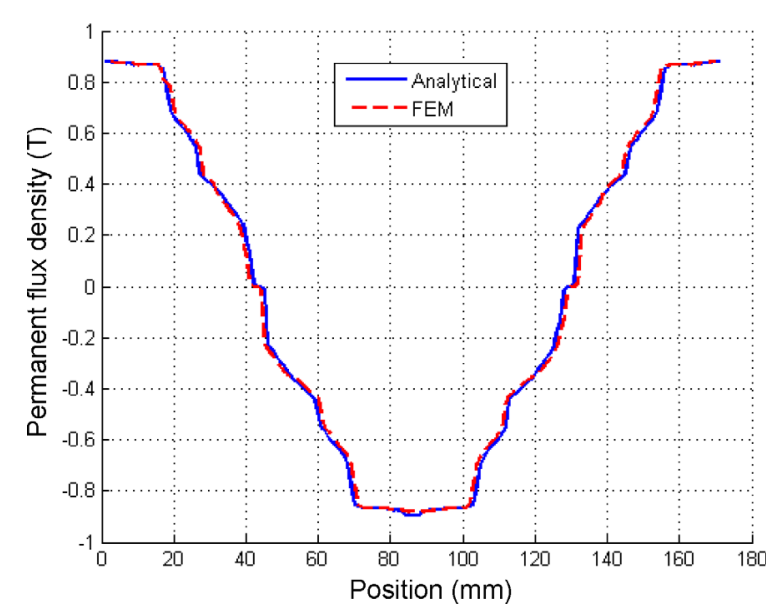

Fig. 7. (Color online) Comparison between the analytically predicted and FE methods calculated distributions of the flux density.

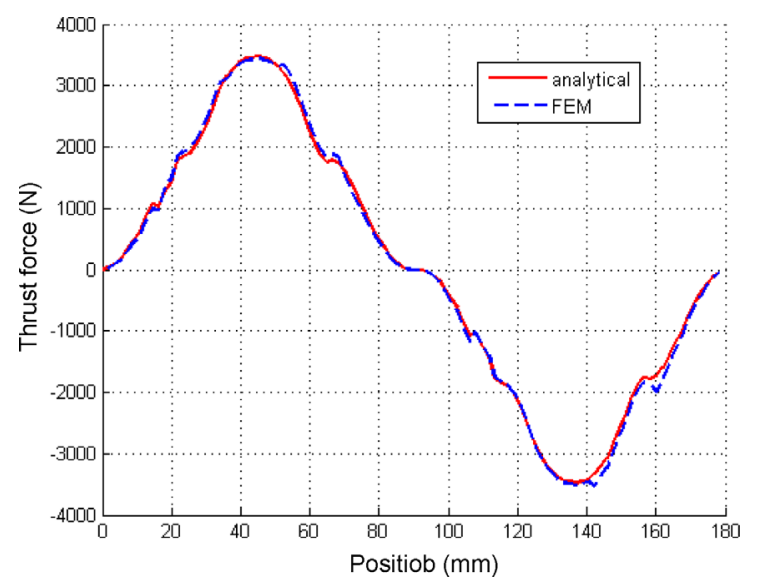

Fig. 8. (Color online) Comparison of analytically and FE methods-predicted thrust force waveforms.

A comparison between the analytically predicted and FE methods calculated distributions of the flux density component, as a function of $z$ position in dual quasiHalbach array, is shown in Fig. 7. A slight difference was observed between the two methods.

Figure 8 compares the analytical and FE predicted thrust force in dual quasi-Halbach array. As shown, the analytically predicted thrust force waveforms agree well with those deduced from the FE methods analysis.

\subsection{FE Optimization of Dual Quasi-Halbach Array PMTG}

In this study, the first FE methods are utilized for designing optimization rectangular shaped PMs, and then FE methods are carried out to design optimized trapezoidal shaped PMs.

Figure 9(a) shows the Quasi-Halbach magnetization (a)

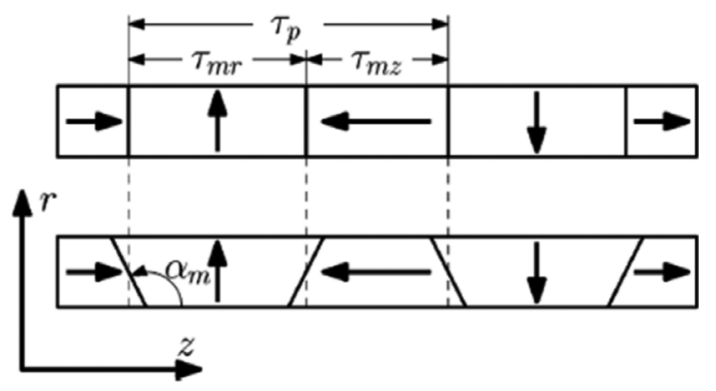

Fig. 9. (Color online) Quasi-Halbach magnetization pattern. (a) Rectangular shaped PMs. (b) Trapezoidal shaped PMs.

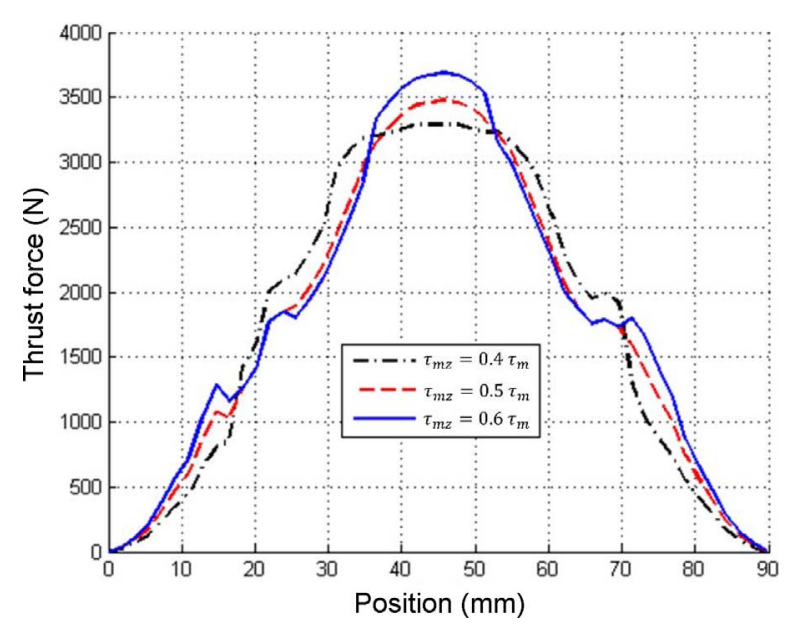

Fig. 10. (Color online) Effect of $\tau_{m z}$ on the waveform of the Radial component of the thrust force in the air gap.

pattern with rectangular shaped PMs, and Fig. 9(b) shows the Quasi-Halbach magnetization pattern with Trapezoidal shaped PMs.

\subsubsection{RECTANGULAR SHAPED PMs}

In this section, this model describes the merits of

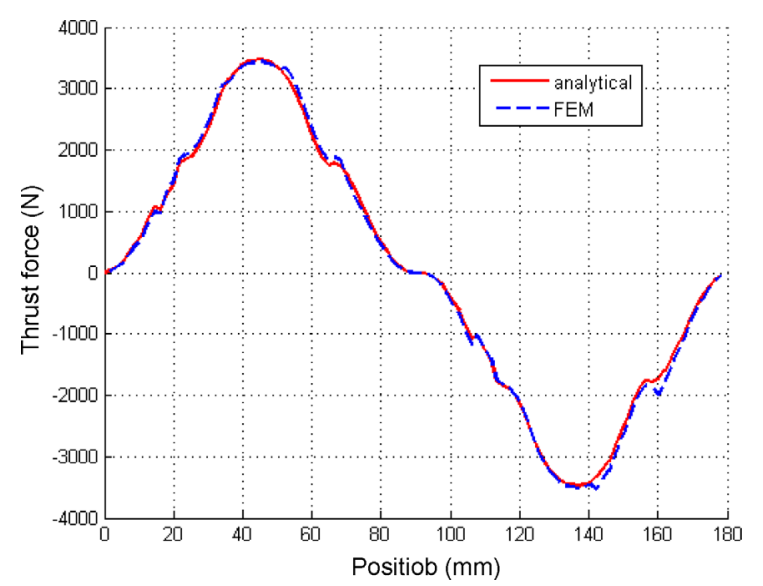

Fig. 11. (Color online) Effect of $\tau_{m z}$ on the waveform of the radial component of the flux density in the air gap. 
Table 1. Variation in the performance parameters with the change in horizontal magnetized magnet proportion.

\begin{tabular}{cccccccc}
\hline \hline $\begin{array}{r}\tau_{m r} \\
(\%)\end{array}$ & $\begin{array}{c}\tau_{m z} \\
(\%)\end{array}$ & $\begin{array}{c}\text { Average Force } \\
(\text { K.N) }\end{array}$ & $\begin{array}{c}\text { Peak Force } \\
(\text { K.N) }\end{array}$ & $\begin{array}{c}\text { Force Ripple } \\
(\%)\end{array}$ & $\begin{array}{c}\text { Average Flux } \\
\text { density (T) }\end{array}$ & $\begin{array}{c}\text { Peak Flux } \\
\text { density (T) }\end{array}$ & $\begin{array}{c}\text { Flux density } \\
\text { Ripple (\%) }\end{array}$ \\
\hline 60 & 40 & 1.78 & 3.29 & 15.84 & 0.69 & 0.87 & 14.85 \\
50 & 50 & 1.72 & 3.47 & 20.13 & 0.66 & 0.91 & 17.67 \\
40 & 60 & 1.67 & 3.68 & 23.92 & 0.64 & 0.94 & 19.04 \\
\hline
\end{tabular}

changing the rectangular shaped PM in Halbach arrays. The variation in the developed thrust force pattern, with the change in proportion of the vertical magnetized magnet $\left(\tau_{m r}\right)$ and the horizontal magnetized magnet, $\left(\tau_{m z}\right)$ is shown in Fig. 10.

Here, the ripple and peak thrust forces increase and average force decrease with increasing proportion of the horizontal magnetized magnet to the vertical magnetized magnet. The flux density distribution in the air gap was calculated for the variation in the vertical magnetized magnet and the horizontal magnetized magnet. Figure 11 shows the magnetic flux density in the air gap of the rectangular shaped PMs. The variation in the peak value, average value, and ripple for the thrust force and flux density of PMTG is listed in Table 1.

You are kindly advised to use this template for editing your submission, as you have nothing to change in terms of paper and text format. Simply applying the styles defined here will be sufficient.

\subsubsection{TRAPEZOIDAL-SHAPED PMs}

In this section, this model is extended to be able to investigate the effect of the angle $\alpha_{m}$ of the trapezoidal magnet on the thrust force and flux density. In order to increase the average thrust force and decrease the ripple, the vertical magnetized magnet is increased to the horizontal magnetized magnet $\left(\tau_{m r}=0.6 \tau_{m}\right)$. The flux density distribution in the air gap and thrust force were calculated for several values of $\alpha_{m}$. Figure 12 shows a comparison among the simulation results of the thrust force of the three proposed designs.

The amplitudes of the thrust force of the PMTG follow the higher amplitude obtained for the proposed design $\alpha_{\mathrm{m}}$ $=75$; however, the average value of the thrust force in $\alpha_{m}$ $=105$ is higher than other cases.

Figure 13 shows a comparison of the simulation results

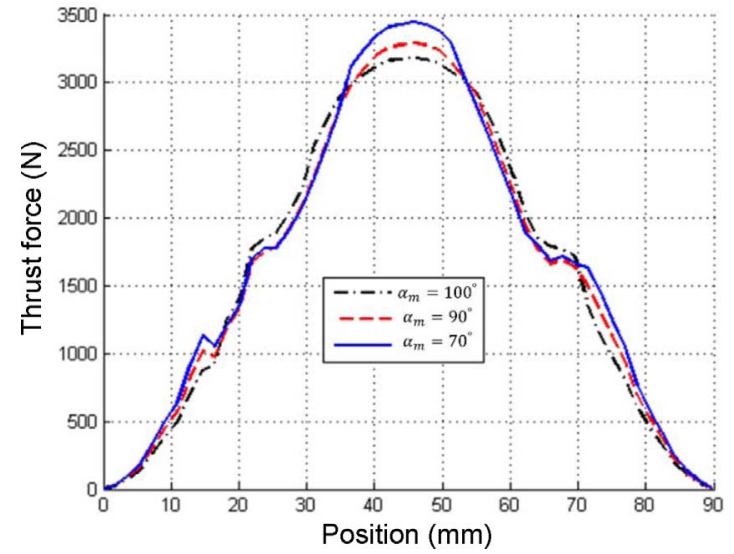

Fig. 12. (Color online) Effect of $\alpha_{m}$ the waveform of the Radial component of the thrust force in the air gap.

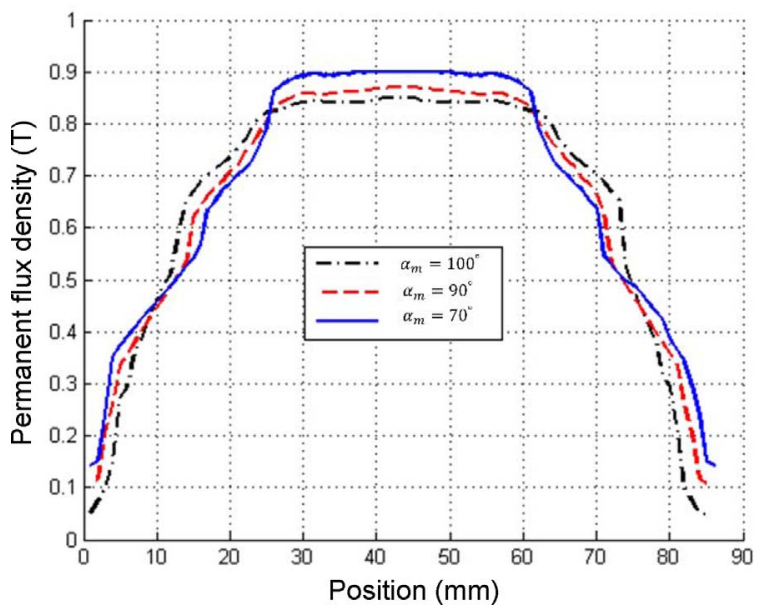

Fig. 13. (Color online) Effect of $\alpha_{m}$ the waveform of the Radial component of the flux density in the air gap.

of the permanent flux density for the three proposed designs. The variation in the peak value, average value, and ripple for the thrust force and flux density of the

Table 2. Variation In The performance parameters with the change in $\alpha_{m}$ proportion.

\begin{tabular}{cccccccc}
\hline \hline $\begin{array}{c}\alpha_{m} \\
(\text { degree })\end{array}$ & $\begin{array}{c}\text { Average Force } \\
(\text { K.N) }\end{array}$ & $\begin{array}{c}\text { Peak force } \\
(\text { K.N })\end{array}$ & $\begin{array}{c}\text { Force Ripple } \\
(\%)\end{array}$ & $\begin{array}{c}\text { Average Flux density } \\
(\mathrm{T})\end{array}$ & $\begin{array}{c}\text { Peak Flux density } \\
(\mathrm{T})\end{array}$ & $\begin{array}{c}\text { Flux density Ripple } \\
(\%)\end{array}$ \\
\hline 105 & 1.80 & 3.18 & 13.46 & 0.70 & 0.85 & 13.28 \\
90 & 1.78 & 3.29 & 15.13 & 0.69 & 0.87 & 14.85 \\
75 & 1.75 & 3.44 & 17.11 & 0.68 & 0.90 & 15.81 \\
\hline
\end{tabular}




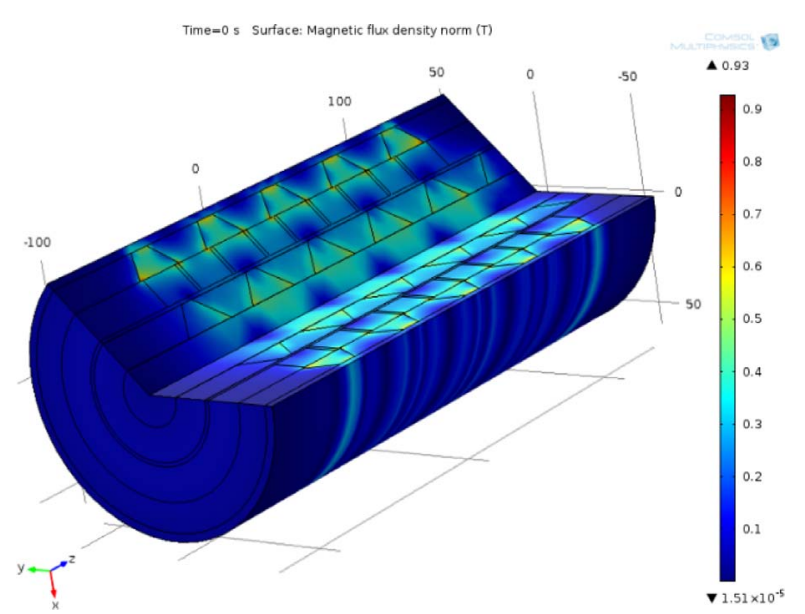

Fig. 14. (Color online) 3D FE methods investigation of the dual quasi-Halbach-array PMTG.

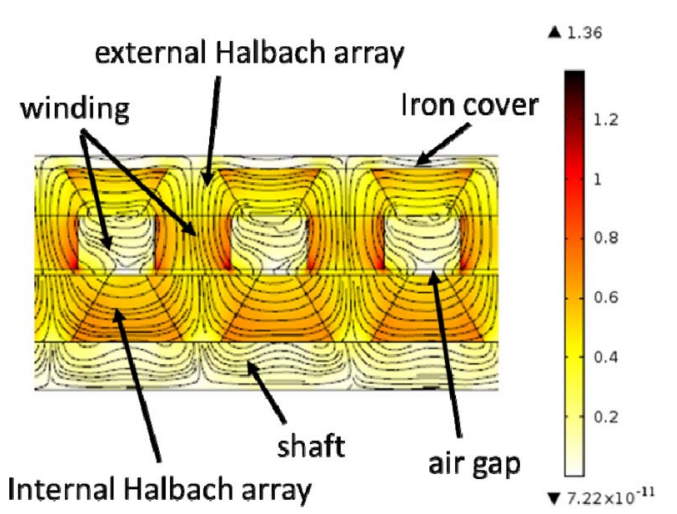

(a)

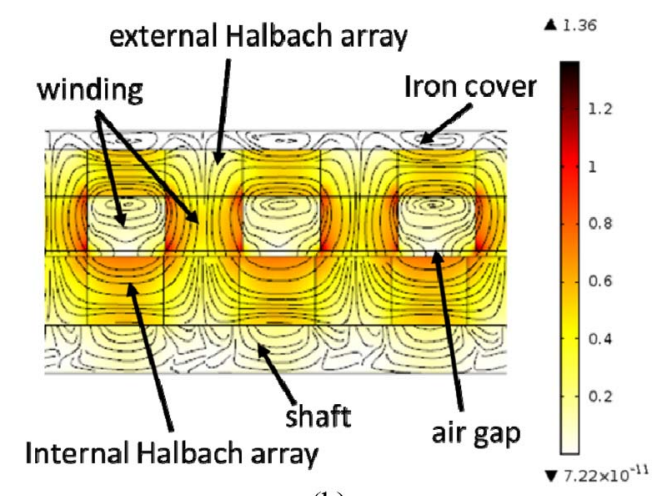

(b)

Fig. 15. (Color online) Flux distributions in generator (a) Trapezoidal topology (b) Rectangular topologiy.

PMTG is listed in Table 2.

As shown, the average thrust force increases and ripple force decreases with increasing proportion of the vertical magnetized magnet to the horizontal magnetized magnet.

The FE model of the designed dual quasi-Halbach-array PMTG in $\tau_{m r}=0.6 \tau_{m}$ and $\alpha_{m}=105^{\circ}$ is shown in Fig. 14 .

According to the results obtained from the FE methods, the trapezoidal topology for $\alpha_{m}=120^{\circ}$ has more uniformity and lower flux leakage than the rectangular one, thus decreasing the ripple force $(1.57 \%)$ and increasing the average flux density in the air gap. Consequently, the electrical characteristic of the generator improved. Figure 15 demonstrates the distribution of the flux for the trapezoidal and rectangular topologies.

\section{Output Power}

To obtain output power for the PMTG, the FE methods were simulated by moving the mesh techniques [14]. The motion of the translator was modeled by moving translator mesh without any modification to the mesh topology. The model reciprocating motion mover was simulated by the force balance equation, as shown in Equation (25) and repeated here as Equation (25) over one complete stroke [13].

$$
A_{B} P_{1} \leq\left(\frac{2 r}{r+1}\right)^{n}\left[\left(1+\frac{x}{x_{m}}\right)^{-n}-\left(1+\frac{x}{x_{m}}\right)^{-n}\right]=m \ddot{x},
$$

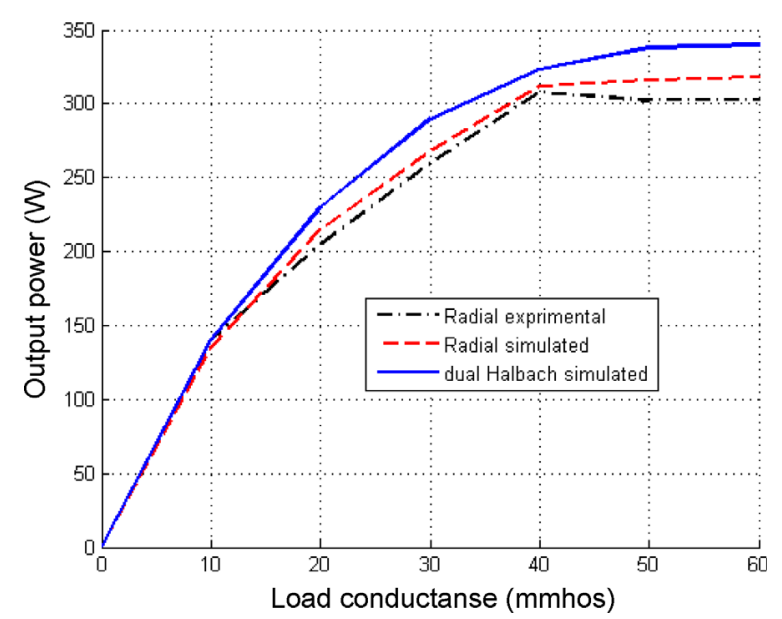

Fig. 16. (Color online) Comparison of output power radial array and dual quasi-Halbach array topology.

Table 3. Generator parameters

\begin{tabular}{cccc}
\hline \hline Number of active rotor poles & $P$ & 4 & Poles \\
\hline Pole pitch & $\tau$ & 4.191 & $\mathrm{Mm}$ \\
Diameter & $D$ & 6.2865 & $\mathrm{Mm}$ \\
Number of windings & $N_{s}$ & 296 & Turns \\
Magnet Height & $h_{m}$ & 18.415 & $\mathrm{Mm}$ \\
Magnet width & $l_{w}$ & 41.0718 & $\mathrm{Mm}$ \\
Magnet Gap & $W_{m g}$ & 0.4191 & $\mathrm{Mm}$ \\
Air Gap & $g$ & 0.1651 & $\mathrm{Mm}$ \\
Magnet Inner Diameter & $I D_{m}$ & 2.6035 & $\mathrm{Mm}$ \\
Magnet outer Diameter & $O D_{m}$ & 6.2865 & $\mathrm{Mm}$ \\
\hline
\end{tabular}


where $A_{B}$ is the bore area; $P_{1}$ is the input intake pressure, $r$ is the compression ratio; $n$ is the ratio of specific heats; $X$ is the translator position; $X_{m}$ is the maximum half stroke; and $m$ is the mass of the translator.

The load testing was conducted using a purely resistive variable load. Figure 16 shows the experimental and simulated generator output power used in the experimental of the generator and the geometric parameters [13]. The geometric parameters of the generator are listed in Table 3 . The engine-generator system was capable of supplying a peak power of $315 \mathrm{~W}$ for radial array and $340 \mathrm{~W}$ for dual quasi-Halbach array.

\section{Conclusions}

In this study, a modest attempt was performed to design a novel dual quasi-Halbach-array PMTG in reciprocating free piston generator system. An analysis based on the Maxwell equations was derived to predict the air gap magnetic flux density distribution and thrust force. The optimization of the machine was performed by the FE methods. The trapezoidal dual quasi-Halbach array magnet configuration helps in achieving high thrust force, increasing output power, and uniform flux density along the air gap of the machine, thereby decreasing the thrust force ripple. The designed machine is simulated, and the results obtained from the analytical calculations were validated with those of the FE methods.

\section{References}

[1] S.-M. Jang and J.-Y. Choi, Journal of Electrical Engineering \& Technology 2, 221 (2007).

[2] J. Wang, M. West, D. Howe, Hector Zelaya-De La Parra, and W. M. Arshad, IEEE Trans. Energy Convers. 22, 299 (2007).

[3] J. Wang and D. Howe, IEEE Trans. Magn. 41, 2479 (2005).

[4] W. H. Arof, and H. W. Ping, IET Electr. Power Appl. 4, 629 (2010).

[5] J. Faiz1, B. Rezaeealam1, and S. Yamada, IEEE Trans. Magn. 42, 2172 (2006).

[6] J. Pan, Y. Zou, and G. Cao, IET Renew. Power Gener, 7, 98 (2013).

[7] W. M. Arshad, Ph.D. dissertation, Royal Inst. Technol., Stockholm, Sweden, 91-7283-535-4 (2003).

[8] S.-M. Jang, S.-H. Lee, and I.-K. Yoon, IEEE Trans. Magn. 38, 3261 (2002).

[9] J. Wang, D. Howe, and G. W. Jewell, IEEE Trans. Magn. 39, 3517 (2003).

[10] L. Yan and L. Zhang, Progress In Electromagnetics Research 136, 283 (2013).

[11] J. Wang and D. Howe, IEEE Trans. Magn. 41, 2470 (2005).

[12] M. Abramowitz and I. A. Stegun, National Bureau of Standards, Washington, DC, 10th Printing, 496 (1972).

[13] W. R. Cawthorne, P. Famouri, J. Chen, N. N. Clark, T. I. McDaniel, R. J. Atkinson, S. Nandkumar, C. M. Atkinson, and S. Petreanu, IEEE Trans. Veh. Technol. 48, 1797 (1999).

[14] S. L. Ho, N. Chen, and W. N. Fu, IEEE Trans. Magn. 47, 2947 (2011). 